\title{
USING Q-CALCULUS TO STUDY LDLT FACTORIZATION OF A CERTAIN VANDERMONDE MATRIX
}

\author{
AleXey KuZnetsov
}

Abstract. We use tools from q-calculus to study $L D L^{T}$ decomposition of the Vandermonde matrix $V_{q}$ with entries $v_{i, j}=q^{i j}$. We prove that the matrix $L$ is given as a product of diagonal matrices and the lower triangular Toeplitz matrix $T_{q}$ with elements $t_{i, j}=1 /(q ; q)_{i-j}$, where $(z ; q)_{k}$ is the q-Pochhammer symbol. We investigate some properties of the matrix $T_{q}$, in particular, we compute explicitly the inverse of this matrix.

Mathematics subject classification (2010): Primary 15A23, Secondary 15B05.

Keywords and phrases: Vandermonde matrix, LU decomposition, Toeplitz matrix, q-Binomial Theorem, q-Pochhammer symbol, discrete Fourier transform.

\section{REFERENCES}

[1] G. E. Andrews, R. Askey, And R. Roy, Special functions, The University Press, Cambridge, 1999.

[2] H. ORUC, Factorization of the Vandermonde matrix and its applications, Applied Mathematics Letters 20 (9): 982-987, 2007.

[3] H. ORUC AND H. K. AKMAZ, Symmetric functions and the Vandermonde matrix, Journal of Computational and Applied Mathematics 172 (1): 49-64, 2004.

[4] H. ORUC AND G. M. Phillips, Explicit factorization of the Vandermonde matrix, Linear Algebra and its Applications 315 (1-3): 113-123, 2000.

[5] C. VAN LOAN, Computational Frameworks for the Fast Fourier Transform, Society for Industrial and Applied Mathematics, 1992. 